\title{
Adenoma pleomorfo del tabique nasal. Caso clínico
}

\author{
Pleomorphic adenoma of the nasal septum: A case report
}

Jaime Osorio $\mathbf{M}^{1}$, Maritza Rahal $\mathrm{E}^{\mathbf{1}}$, Daniel Cantero $\mathbf{C}^{\mathbf{1}}$, Jorge Castillo $\mathrm{A}^{2}$.

\begin{abstract}
RESUMEN
El adenoma pleomorfo es la neoplasia más frecuente de glándulas salivales mayores, pero es poco común en la cavidad nasal. Se presenta el caso clínico de una mujer de 41 años, quien consulta por una masa septal izquierda que determina obstrucción nasal ipsilateral cuyo aspecto clínico e imagenológico sugiere una lesión benigna. Se extirpa la lesión por abordaje transnasal directo, con diagnóstico histopatológico compatible con adenoma pleomorfo del tabique nasal (tumor mixto benigno de tipo salival). Se analiza el caso de adenoma pleomorfo de cavidad nasal y se efectúa una revisión bibliográfica del tema. Su importancia radica en su consideración en el diagnóstico diferencial de neoplasias que se desarrollan en la nariz o cavidades perinasales.
\end{abstract}

Palabras clave: Adenoma pleomorfo, tabique nasal.

\begin{abstract}
Pleomorphic adenoma is the most frequent major salivary glands neoplasm but it is rare in the nasal cavity. We report a case of a 41 years old woman who complains of a left septal mass and unilateral nasal obstruction. Her clinical and CT-scan aspects suggest a benign lesion. The lesion is removed by direct transnasal approach. The histopathological diagnosis informed pleomorphic adenoma of the nasal septum (benign mixed tumor of salivary-type). We review the literature of nasal pleomorphic adenoma. This diagnosis should be considered in the differential diagnosis of tumors that develop in the nose or perinasal cavities.
\end{abstract}

Key words: Pleomorphic adenoma, nasal septum.

Médico del Servicio de Otorrinolaringología, Hospital Barros Luco Trudeau.

2 Médico del Servicio de Anatomía Patológica, Hospital Barros Luco Trudeau. 


\section{INTRODUCCIÓN}

Los tumores de glándulas salivales constituyen alrededor del $3 \%$ de todas las neoplasias y el $1 \%$ a $6 \%$ de los de cabeza y cuello.

El adenoma pleomorfo es la neoplasia benigna más frecuente de las glándulas salivales ${ }^{1-3}$. El primero en describir este tipo de tumor de glándula salival, caracterizado por un componente epitelial con zonas de diferenciación glandular, rodeado de un estroma variable, fue Billroth en 1859. Posteriormente Willis, en 1967, sugirió el nombre de adenoma pleomorfo debido al patrón histológico poco usual que lo caracteriza, pues exhibe tejido epitelial entremezclado con diferentes tejidos mesenquimatosos $\mathrm{s}^{4-6}$.

Las glándulas salivales menores son afectadas por adenoma pleomorfo en sólo $8 \%$ a $11 \%$ de los casos. Se sabe que mientras más pequeña es la glándula salival, es menos probable que se trate de un tumor benigno. El adenoma pleomorfo intranasal es extremadamente raro, encontrándose reportes de tres series, junto con casos aislados ${ }^{5-10}$. En la literatura nacional no hay casos publicados en nariz 0 cavidades perinasales.

El objetivo es publicar un caso y revisar la literatura al respecto.

\section{CASO CLÍNICO}

Mujer de 41 años de edad con antecedentes de hipertensión arterial crónica, quien consulta por la aparición de un tumor en la fosa nasal izquierda, ubicada a $1 \mathrm{~cm}$ del borde narinario, de dos años de evolución, de crecimiento lento llegando a obstruir la fosa nasal con deformación del ala nasal izquierda y epistaxis leve en algunas ocasiones (Figuras 1 y 2).

Se realiza tomografía computarizada de nariz y cavidades perinasales que muestra en la fosa nasal izquierda, en su porción anterior, ocupación de la vía aérea por una masa redondeada con base en el tabique nasal, que capta tenuemente el contraste, abomba discretamente el ala de la nariz y con características de lesión benigna (Figura 3).

Se extirpa dicha lesión por vía transnasal directa incluyendo la mucosa nasal subyacente y alcanzando hasta el plano cartilaginoso.

Se envía a estudio histopatológico, un nódulo blanquecino, de consistencia dura, de $2 \mathrm{~cm}$ de diámetro, y la mucosa resecada que mide 3,5 por $1,9 \mathrm{~cm}$ (Figuras 4 y 5$)$.

En el estudio histopatológico se identifica mucosa nasal con componente inflamatorio, una pseudocápsula fibrosa que envuelve un tumor y septos fibrosos (Figura 6). En la Figura 7, se observa que el tumor está constituido de izquierda a derecha por elementos epiteliales, mioepiteliales y estromales. El componente epitelial conforma elementos glandulares, las células mioepiteliales son redondeadas y en menor cuantía fusiformes (Figura 8). No se evidencian elementos de malignidad ni de infiltración.

El diagnóstico final es de adenoma pleomorfo (tumor mixto benigno de tipo salival) del tabique nasal izquierdo, con bordes quirúrgicos libres, sin evidencia de elementos de malignidad.

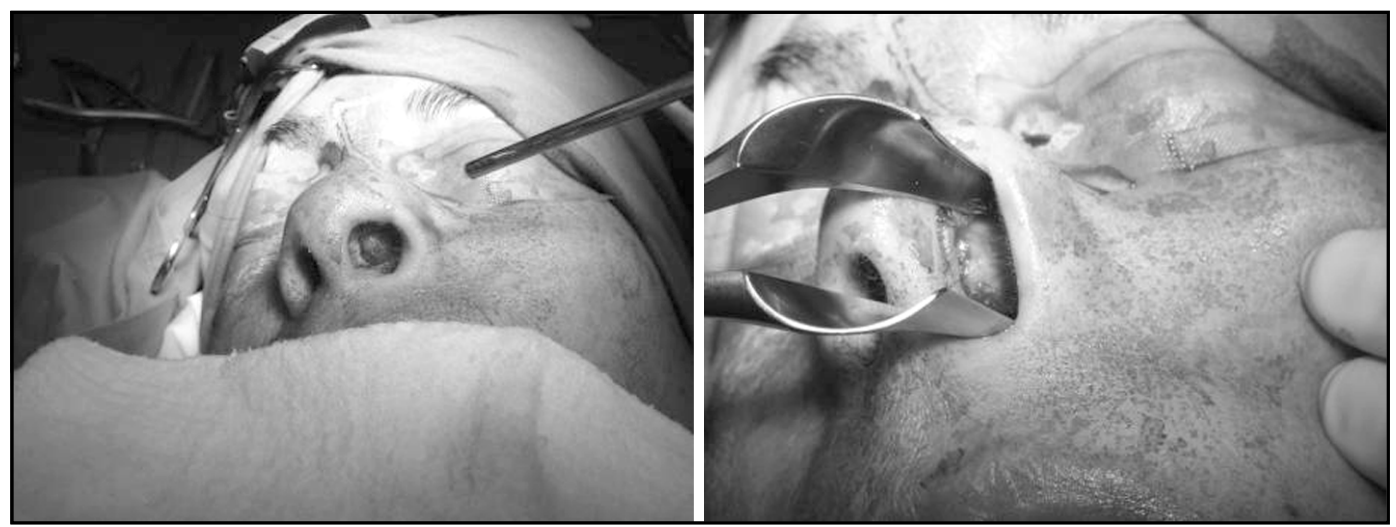

Figuras 1 y 2. Tumor nodular con base de implantación ancha a $1 \mathrm{~cm}$ del borde narinario, en la fosa nasal izquierda, que deforma el ala de la nariz. 


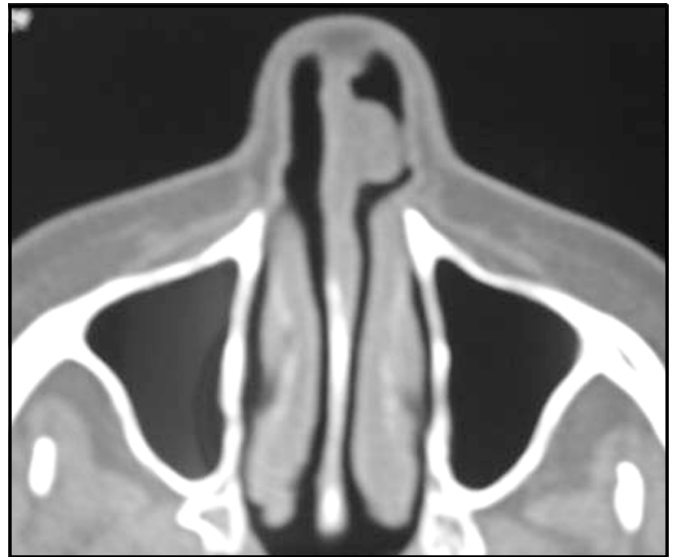

Figura 3. Corte axial de tomografía computarizada de cavidades perinasales con lesión en fosa nasal izquierda correspondiente a adenoma pleomorfo del tabique nasal.

La evolución posquirúrgica es satisfactoria, con adecuada reepitelización de la zona cruenta septal. No presenta complicaciones. La paciente se encuentra asintomática y libre de enfermedad a los cinco meses de la cirugía.

\section{DISCUSIÓN}

El primer caso reportado en la literatura fue publicado por Denker en 19291.

Los adenomas pleomórficos constituyen la mayoría de los tumores benignos de las glándulas salivales menores. Se han reportado casos en cavidad nasal, cavidades perinasales, naso-

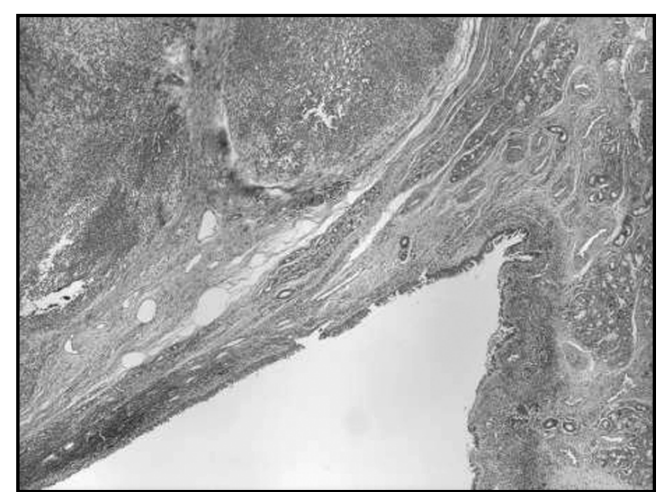

Figura 6. Corte histológico de adenoma pleomorfo de tabique nasal, tinción de hematoxilina y eosina, aumento 4x.

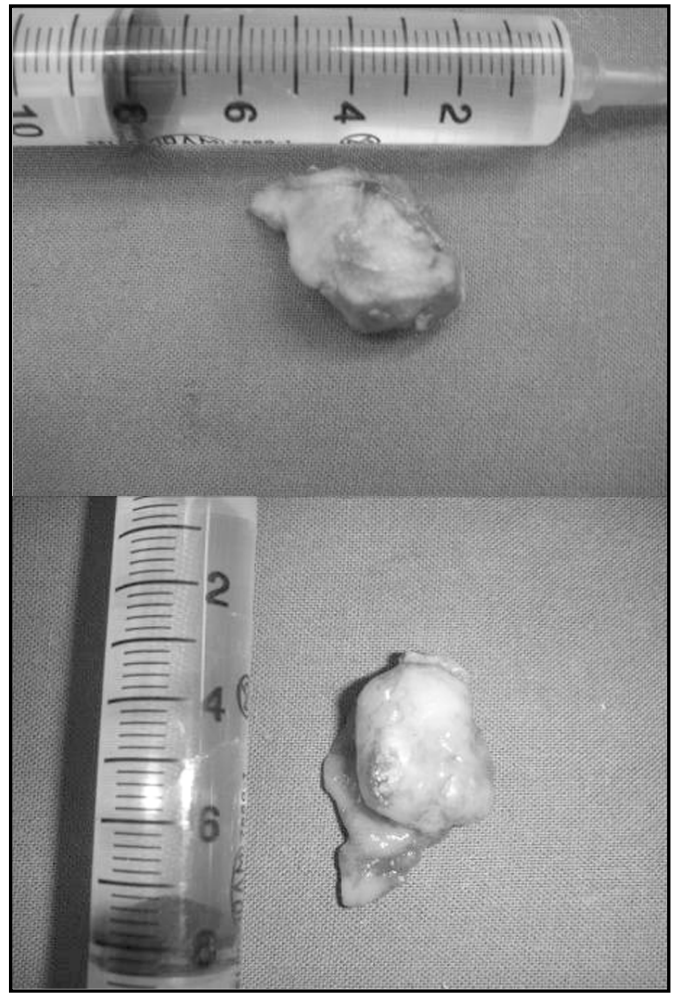

Figuras 4 y 5 . Lesión nodular de tabique nasal extirpada. La muestra corresponde a adenoma pleomorfo y mucosa subyacente.

faringe, orofaringe, hipofaringe y laringe. Dentro de las neoplasias de fosa nasal, el adenoma pleomorfo constituye un diagnóstico poco frecuente ${ }^{2,7}$

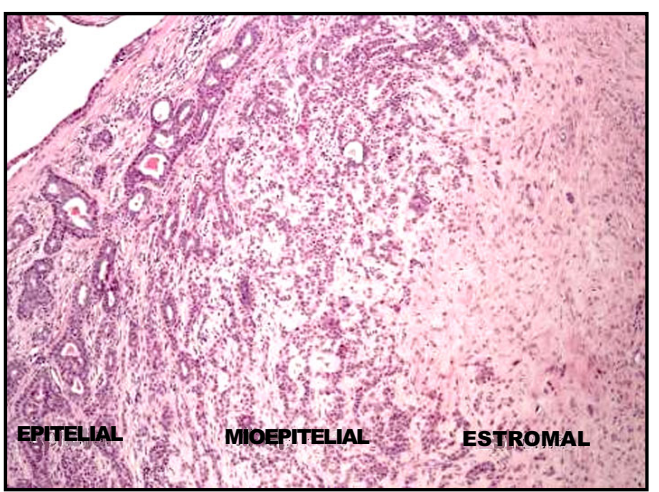

Figura 7. Corte histológico de adenoma pleomorfo de tabique nasal, tinción de hematoxilina y eosina, aumento 10x. 


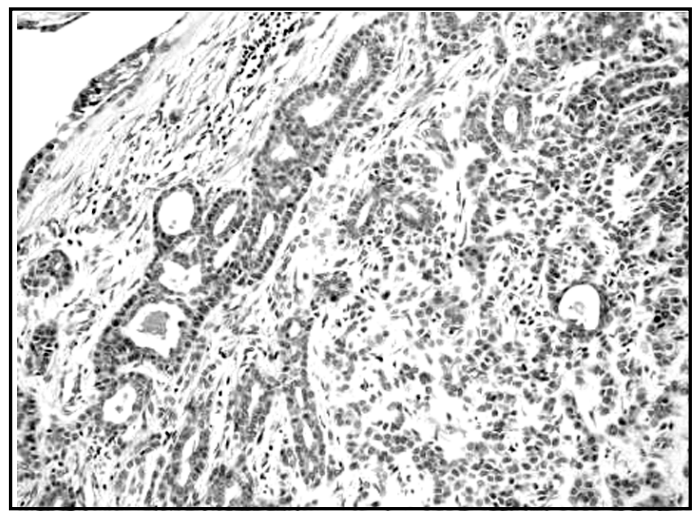

Figura 8. Corte histológico de adenoma pleomorfo de tabique nasal, tinción de hematoxilina y eosina, aumento 20x.

Según Spiro, la frecuencia de presentación del adenoma pleomorfo en glándula salival menor en el tracto respiratorio superior, de mayor a menor es: paladar, labios, cavidad nasal, lengua, orofaringe, nasofaringe, lo que concuerda con la densidad de glándulas salivales menores en esas regiones?

Aunque la mayoría de las glándulas salivales menores en la cavidad nasal se encuentran en la pared lateral de la fosa nasal, la mayoría de los adenomas pleomórficos se localizan en el tabique nasal $(80 \%)^{8-10}$. Esto ha generado una controversia en cuanto a su origen, ya que Matthew ${ }^{5}$ plantea su origen en células epiteliales ectodérmicas embrionarias aberrantes. Stevenson ${ }^{5}$, a su vez, propone que se originarían de posibles restos embriológicos del órgano vomeronasal de Jacobson. Evans y Cruickshank ${ }^{5}$ opinan que los tumores mixtos son de origen enteramente epitelial, originándose en tejido de glándula salival plenamente desarrollado y no en restos embrionarios, planteamiento que se acepta mayormente en la actualidad 5 .

El adenoma pleomórfico de cavidad nasal se presenta entre la $3^{\mathrm{a}}$ y la $6^{\mathrm{a}}$ década de la vida con mayor frecuencia en edades cercanas a los 40 años, hecho representado en nuestro caso clínico. Según Suzuki, tendrían una preponderancia por el género femenino, tendencia que no comparte Compagno $0^{5-6}$.

La presentación clínica se caracteriza en $71 \%$ a $75 \%$ por obstrucción nasal y en $56 \%$ por epistaxis. Los síntomas están dados por el tamaño tumoral ya que podemos encontrar pacientes asintomáticos con una masa intranasal hasta otros con deformación de la pirámide nasal. En general, el crecimiento del adenoma es lentamente progresivo, de semanas a años ${ }^{8,9}$.

El tamaño del tumor puede variar entre $5 \mathrm{~mm}$ a $7 \mathrm{~cm}$. La descripción clínica típica es la de un tumor exofítico, polipoideo, ovalado, con forma de cúpula, otras con aspecto irregular, nodular 0 de masa gris firme. A menudo se presenta cubierto con una membrana o una mucosa translúcida. En la cavidad nasal se expresa generalmente como una masa de forma polipoidea de amplia base de implantación con pseudocápsula $a^{4,5,8,11}$.

Histológicamente se reconocen las características típicas de un adenoma pleomórfico, pero en la cavidad nasal, presentan mayor celularidad con predominio de los elementos epiteliales y mioepiteliales frente a los estromales. Las células epiteliales pequeñas ovaladas a menudo se disponen en masa 0 cordones densamente poblados. Raramente, los estromas mixoides, condroides 0 con colágeno predominan como en el tumor mixto tradicional de las glándulas salivales mayores 5 . Así, la característica microscópica más notable de los tumores mixtos intranasales es su mayor celularidad reflejando una composición predominantemente epitelial ${ }^{5}$.

La inmunohistoquímica encontrada en los adenomas pleomórficos de cavidad nasal es similar a la de los de glándula parótida. Son positivas las citoqueratinas para componente epitelial y actina, y S-100 para células mioepiteliales ${ }^{4,8}$. Debe ser usada en caso de duda ya 
que normalmente la microscopía simple nos permite hacer el diagnóstico.

El diagnóstico diferencial se puede establecer con cualquier neoplasia de cavidad nasal como papiloma, angioma, carcinoma escamoso, carcinoma adenoideo quístico, adenocarcinoma, glioma, meningioma, estesioneuroblastoma, condrosarcoma, leiomioma, hemangiopericitoma, oncocitoma y otros ${ }^{9}$. Dentro del diagnóstico diferencial se debe recordar el granuloma piógeno o inflamatorio que en la práctica clínica es bastante frecuente.

Los hallazgos en la tomografía computarizada (TC) no son específicos, identificándose una imagen bien definida sugerente de masa de tejido blando en la cavidad nasal. Estas lesiones se asocian con la remodelación ósea progresiva suave de los huesos que rodean, lo que indica un curso indolente. La calcificación es rara. La remodelación ósea suave y la falta de calcificación sugieren un crecimiento lento y un proceso benigno en lugar de uno neoplásico maligno agresivo ${ }^{12}$.

La resonancia magnética $(\mathrm{RM})$ parece ser una herramienta de diagnóstico más fiable que la TC para el diagnóstico de adenoma pleomórfico intranasal, porque es posible evaluar la relación del tumor con las estructuras adyacentes en varios planos anatómicos. Los adenomas pleomorfos generalmente son heterogéneos, con señal de intensidad baja a intermedia en $\mathrm{T} 1 \mathrm{e}$ intensidad alta e intermedia en T2. Una cápsula hipointensa puede verse en imágenes ponderadas en T211,12. En nuestro medio, debido a su mayor costo, el estudio con resonancia se justifica solamente en caso de sospecha de una neoplasia maligna.

Se han descrito adenomas pleomorfos nasales con diferenciación de músculo esquelétic $0^{13}$, neoformación osteoide ${ }^{14}$, transformación maligna e incluso adenomas pleomorfos nasales metastizantes ${ }^{15,16}$.

La resección local con márgenes histológicos libres es generalmente adecuado como tratamiento de elección para tumores de glándulas salivales ${ }^{1,4}$. El abordaje elegido para la extirpación dependerá del tamaño y la localización; pudiendo incluir resección transnasal endoscópica 0 directa, degloving hemifacial y rinotomía lateral. La transnasal es la más eficaz y menos agresiva, así como la más utilizada actualmente. La extirpación septal y disección cervical está reservada para tumores metastizantes ${ }^{4,6}$.
La tasa de recidiva del adenoma pleomórfico septal es más baja (menos del 10\%) que la tasa del mismo tumor en glándula parótida $(50 \%)$ e intraoral $(25 \%)$. Esta baja recidiva se explicaría por el bajo porcentaje de células estromales 0 mesenquimáticas que son las que contribuirían a aumentar la recurrencia o por la facilidad para obtener márgenes libres en comparación con otras Iocalizaciones ${ }^{4-6}$.

En suma, el adenoma pleomórfico de cavidad nasal es infrecuente y, por lo general, es similar al tumor mixto de las glándulas salivales mayores pero, difiere en su mayor grado de celularidad, una menor tasa de recurrencia, y menor agresividad biológica. Si bien es una lesión benigna, ésta debe ser considerada como diagnóstico diferencial de neoplasia maligna en la cavidad nasal, fundamentalmente cuando un paciente refiere obstrucción nasal unilateral y/o epistaxis como motivo de consulta.

\section{BIBLIOGRAFÍA}

1. Gana P, Masterson L. Pleomorphic adenoma of the nasal septum: a case report. J Med Case Reports 2008; 2: 349-51.

2. Celedón C, Ojeda JP, Agurto M, Olavarría C, et al. Tumores de glándulas salivales. Experiencia de 20 años. Rev Otorrinolaringol Cir Cabeza Cuello 2002; 62: 255-64.

3. Castillo h, Plandiura J, Calabresse R. Tumores de glándulas salivales menores, revisión de diez años. Rev Otorrinolaringol Cir Cabeza CueIlo 1998; 58: 83-7.

4. Tahlan a, Nanda $A$, Nagarkar $N$, Bansal S. Pleomorphic adenoma of the nasal septum: a case report. Am J Otolaryngol 2004; 25: 11820.

5. Compagno J, Wong RT. Intranasal mixed tumors (pleomorphic adenomas). A clinicopathologic study of 40 cases. Am J Clin Pathol 1977; 68: 213-8.

6. Suzukı K, Moribe K, Baba S. Abstract: A rare case of pleomorphic adenoma of lateral wall of nasal cavity, with special reference of statistical observation of pleomorphic adenoma of nasal cavity in Japan. Nippon Jibiinkoka Gakkai Kaiho 1990; 5: 740-5. 
7. Spiro RH, Koss LG, Hajdu SI, et al. Tumors of minor salivary origin. A clinicopathologic study of 492 cases. Cancer 1973; 31: 117-29.

8. Jassar P, Stafford ND, Macdonald aW. Pleomorphic adenoma of the nasal septum. $J$ Laryngol Otol 1999, 113 (5): 483-5.

9. Jackson L, Rosenberg S. Pleomorphic adenoma of the lateral nasal wall. Otolaryngol Head Neck Surg 2002; 127: 474-6.

10. Mackle T, Zahirovic A, Walsh M. Pleomorphic adenoma of the nasal septum. Ann Otol Rhinol Laryngol 2004; 113 (3): 210-1.

11. Oztürk E, Saglam 0, Sönmez $G$, Cüce $F$, Haholu $A$. $\mathrm{CT}$ and $\mathrm{MRI}$ of an unusual intranasal mass: pleomorphic adenoma. Diagn Interv Radiol 2008; 14(4): 186-8.

12. Clark M, Fatterpekar GM, Mukherul SK, Buenting
J. CT of intranasal pleomorphic adenoma. Neuroradiology 1999; 41: 591-3.

13. Lam PW, Chan JK, Sin VC. Nasal pleomorphic adenoma with skeletal muscle differentiation: potential misdiagnosis as rhabdomyosarcoma. Hum Pathol 1997; 28: 1299-302.

14. Lee KC, Chan JK, Chong YW. Ossifying pleomorphic adenoma of the maxillary antrum. J Laryngol Otol 1992; 106: 50-2.

15. Wenig BM, Hitchcock CL, Ellis GL, Gnepp DR. Metastasizing mixed tumor of salivary glands. A clinicopathologic and flow cytometric analysis. Am J Surg Pathol 1992; 16: 845-58.

16. Freeman SB, Kennedy KS, Parker GS, Tatum SA. Metastasizing pleomorphic adenoma of the nasal septum. Arch Otolaryngol Head Neck Surg 1990; 116: 1331-3. 